\title{
Physical aging effects on the dynamic relaxation behavior and mechanical properties of $\mathrm{Cu}_{46} \mathrm{Zr}_{46} \mathrm{Al}_{8}$ metallic glass
}

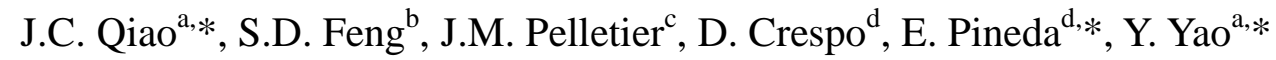 \\ ${ }^{a}$ School of Mechanics, Civil Engineering and Architecture, Northwestern Polytechnical University, \\ $X i$ 'an 710072, China \\ ${ }^{b}$ Advanced Manufacturing Technology Research Centre, Department of Industrial and Systems \\ Engineering, The Hong Kong Polytechnic University, Hong Kong \\ ${ }^{c}$ Université de Lyon, MATEIS, UMR CNRS 5510, Bat. B. Pascal, INSA-Lyon, F-69621 \\ Villeurbanne cedex, France \\ ${ }^{d}$ Departament de Física, Universitat Politècnica de Catalunya, 08860-Castelldfels, Barcelona, \\ Spain
}

(Submitted to Journal of Alloys and Compounds)

Revised version: May 17, 2017

*Corresponding author:

Dr. J.C. Qiao

E-mail address: qjczy@ hotmail.com

Dr. E. Pineda

E-mail address: eloi.pineda@upc.edu

Dr. Y. Yao

E-mail address: yaoy@nwpu.edu.cn 


\begin{abstract}
Mechanical relaxation of glassy materials is strongly influenced by their mechanical and physical properties. In the current study, dynamic mechanical relaxation of $\mathrm{Cu}_{46} \mathrm{Zr}_{46} \mathrm{Al}_{8}$ metallic glass has been investigated by mechanical spectroscopy and molecular dynamics simulations. Our results show that mechanical properties of metallic glasses are highly dependent on their thermal history. $\alpha$ relaxation in the frequency region of the $\mathrm{Cu}_{46} \mathrm{Zr}_{46} \mathrm{Al}_{8}$ metallic glass was described by Havriliak-Negami $(\mathrm{HN})$ model. In the framework of the quasi-point defects theory, the correlated factor $\chi$ could be used to characterize the concentration of "defects" of glassy materials. By molecular dynamics simulations of dynamic mechanical tests at different temperatures and frequencies, it is noted that adjusting of defects is inversely correlated to storage modulus at the atomic level. On the other hand, physical aging below the glass transition temperature $T_{g}$ reduces the concentration of defects and narrows down the width of shear bands, which corresponds to decreasing of the atomic mobility.
\end{abstract}

Keywords: Metallic glasses; Mechanical spectroscopy; Physical aging; Mechanical properties 


\section{Introduction}

Metallic glasses have attracted research interests due to their unique physical and mechanical properties, such as large elastic deformation, high strength, excellent thermoplastic formability and corrosion resistance [1-3]. It has been documented that relaxation dynamics is closely related to the mechanical and physical properties of metallic glasses [4-7]. The dynamics of atomic rearrangements in metallic glasses can be investigated by using mechanical relaxation. From mechanical spectroscopy or dynamic mechanic analysis (DMA), the dynamic modulus $\left(\mathrm{G}=\mathrm{G}^{\prime \prime} / \mathrm{G}^{\prime}\right)$ can be obtained, which is composed by the storage $\left(\mathrm{G}^{\prime}\right)$ and loss $\left(\mathrm{G}^{\prime \prime}\right)$ shear moduli. The loss factor (also called internal friction) is defined as $\tan \delta=\mathrm{G}^{\prime \prime} / \mathrm{G}^{\prime}$.

Metallic glasses remain in a non-equilibrium state due to the rapid solidification process. A fundamental aspect with regard to the physics of glass transition for different types of amorphous materials (i.e. amorphous polymers, glassy oxides and metallic glasses) is to study dependence of the material dynamics on the thermodynamic variables. The dynamical properties are generally measured by investigating the relaxation behaviour with techniques such as dielectric and mechanical spectroscopies [7]. Two relaxation processes are typically found in the mechanical spectra: (i) The main relaxation, observed near the glass transition temperature $T_{g}$, is a universal feature of most of the amorphous materials and it is attributed to the cooperative motion of the molecules. The $\alpha$ relaxation of glassy materials is closely related to the glass transition temperature $T_{g}$ and the plastic flow during the deformation [2,4-6]. The characteristic time of $\alpha$ relaxation in glassy materials can be described by the Vogel-Fulcher-Tamman equation [7]:

$\tau_{\alpha}=\tau_{\infty}^{\alpha} \exp \left(\frac{B}{T-T_{0}}\right)$

where $\tau_{\infty}^{\alpha}$ is the relaxation time in the limit of high temperatures, $B$ is a constant and $T_{0}$ represents the Vogel temperature. Previous work has shown that the apparent activation energy of the $\alpha$ relaxation near $T_{g}$ is around $3 \sim 8 \mathrm{eV}$, indicating that the $\alpha$ relaxation is associated with the global and cooperative movements of atoms [8-12]. 
(ii) The Johari-Goldstein (JG or $\beta$ ) relaxation, which is generally detected at lower temperature (or higher frequency). Its intensity is usually much smaller than the main process and it is not evident in all amorphous materials [4-6, 13-15].

In case a well resolved JG relaxation is not observed, it is presented as a shoulder on the low temperature (or high frequency) side of the $\alpha$ relaxation, referred as an “excess wing". In contrast to the $\alpha$ relaxation, $\beta$ relaxation in glassy materials shows Arrhenius-type temperature dependence [7]. It is noted that the activation energy of the $\beta$ relaxation of metallic glasses obeys $U_{\beta} \sim 26 R T_{\mathrm{g}}$ ( $R$ is the gas constant) [4-6]. In addition, $\beta$ relaxation is the precursor of the $\alpha$ relaxation. More recently, additional lower energy relaxation processes were found by analysis to the loss modulus $[16,17]$. These were termed fast $\beta$-or $\beta "$ - relaxation processes in contrast to the already described, slow $\beta$ - or $\beta$ ' - relaxation processes. The typical values of activation energy of the fast $\beta$ relaxation is around $10 \%$ of $\alpha$ relaxation $[16,17]$.

Based on the mechanical relaxation results, it was found by several researchers that the slow $\beta$ relaxation is connected to the physical and mechanical properties in metallic glasses: (i) the $\beta$ relaxation is associated with the plastic deformation [18]; (ii) the activation energy of the slow $\beta$ relaxation is related to the potential energy barriers of the shear transformation zones [19]; (iii) the slow $\beta$ relaxation is linked to the diffusion motion of the smallest constituent atomic species [20] (iv) the slow $\beta$ relaxation depends on the chemical interactions among all the constituting atoms $[21,22]$.

It is well accepted that the physical and mechanical properties of metallic glasses strongly depend on the physical aging or annealing below $T_{g}$. In the current work, we mainly focus on the influence of physical aging below the glass transition temperature $T_{g}$ on the dynamic mechanical behaviour and mechanical properties of $\mathrm{Cu}_{46} \mathrm{Zr}_{46} \mathrm{Al}_{8}$ metallic glass.

\section{Experimental procedures}

$\mathrm{Cu}_{46} \mathrm{Zr}_{46} \mathrm{Al}_{8}$ (at.\%) metallic glass was prepared in a melting equipment under a 
$\mathrm{Zr}$-gettered Ar atmosphere. The master alloy was re-melted 5 times to ensure the chemical homogeneity. Metallic glass plates with a thickness of $1 \mathrm{~mm}$ were obtained by using a copper-mould casting method. The thermal properties of the metallic glass were studied by differential scanning calorimetry (DSC) using a standard commercial instrument (Perkin Elmer, DSC-7) at a heating rate $20 \mathrm{~K} / \mathrm{min}$. The glass transition temperature $T_{g}$ is determined by DSC at $691 \mathrm{~K}$. The amorphous characteristic of the metallic glass was confirmed by X-ray diffraction (XRD).

The dynamic mechanical analysis of the metallic glass was performed on a mechanical spectrometer with an inverted torsion mode, which has been described by Etienne et al [23]. The experiments were carried out under sinusoidal stress, at fixed frequency and constant heating rate, or at a constant temperature by applying various frequencies. The experimental samples had the approximate dimension of $30 \mathrm{~mm}$ (length) $\times 3 \mathrm{~mm}$ (width) $\times 1 \mathrm{~mm}$ (thickness). Isochronal testing was carried out with a constant driving frequency of $0.3 \mathrm{~Hz}$ at a constant heating rate of $3 \mathrm{~K} / \mathrm{min}$. Isothermal experiments were performed under frequency ranges between $10^{-2}$ to $2 \mathrm{~Hz}$. The experiments were carried out in a high vacuum atmosphere and the strain amplitude was lower than $10^{-4}$.

Molecular dynamics (MD) simulations were performed by using LAMMPS [24] with an embedded atom method (EAM) potential[25]. The $\mathrm{Cu}_{46} \mathrm{Zr}_{46} \mathrm{Al}_{8} \mathrm{MGs}$ model, with dimension around $12 \times 12 \times 12 \mathrm{~nm}$, was filled with approximately 100,000 atoms. The model was initially equilibrated for 1 ns with periodic boundary conditions at $2000 \mathrm{~K}$ and $0 \mathrm{~Pa}$. The model was then cooled to a glass state $(100 \mathrm{~K})$ at $1 \mathrm{~K} / \mathrm{ps}$ with $0 \mathrm{~Pa}$ (NPT ensemble). To obtain dynamic deformation information, molecular dynamics simulations of dynamic mechanical spectroscopy (MD-DMS) were employed [26]. This method applies a sinusoidal strain $\varepsilon=\varepsilon_{A} \sin \omega t$, where $\omega$ is the period. The amplitude of $\varepsilon_{A}$ was fixed at $2.5 \%$ within the linear elastic regime. The resultant stress was fitted as $\sigma=\sigma_{A} \sin (\omega t+\delta)$, where $\delta$ represents the phase difference between stress and strain. The storage $\left(E^{\prime}\right)$ and loss $\left(E^{\prime \prime}\right)$ modulus values were calculated as $E^{\prime}=\sigma_{A} / \varepsilon_{A} \cos (\delta)$ and $E^{\prime \prime}=\sigma_{A} / \varepsilon_{A} \sin (\delta)$, respectively. 
Additionally, larger $\mathrm{Cu}_{46} \mathrm{Zr}_{46} \mathrm{Al}_{8}$ metallic glass models with or without annealing treatments were adopted to simulating the compression tests. These simulations had dimensions around $28 \times 5.6 \times 56 \mathrm{~nm}$ filled with approximately 500,000 atoms. Compression tests were achieved by applying a constant and uniaxial strain rate of 4 $\times 10^{7} \mathrm{~s}^{-1}$. Free boundary conditions were applied along the $\mathrm{x}-$ and $\mathrm{z}$-axis, while a periodic boundary condition was applied along the y-axis.

\section{Results and discussion}

Fig.1 displays evolution of the normalized storage modulus $\left(\mathrm{G}^{\prime}\right)$ and loss modulus $\left(\mathrm{G}^{\prime \prime}\right)$ of the $\mathrm{Cu}_{46} \mathrm{Zr}_{46} \mathrm{Al}_{8}$ metallic glass from ambient temperature to $873 \mathrm{~K}$. $G_{u}$ is the non-relaxed modulus, which is assumed to be the value of $\mathrm{G}^{\prime}$ at room temperature. Similar to other metallic glasses [22, 27-29], the mechanical relaxation behaviour of the $\mathrm{Cu}_{46} \mathrm{Zr}_{46} \mathrm{Al}_{8}$ glass shows three different temperature domains based on the storage and loss modulus: (i) In the low temperature range (from the room temperature to $625 \mathrm{~K}$ ), the material is in glassy state and the elastic behavior dominates. In this temperature range, there is no notable $\beta$ relaxation peak of the loss modulus being observed for $\mathrm{Cu}_{46} \mathrm{Zr}_{46} \mathrm{Al}_{8}$. (ii) At intermediate temperatures (from 625 $\mathrm{K}$ to $760 \mathrm{~K}$ ), the storage modulus decreases dramatically and the loss modulus increases simultaneously. This phenomenon corresponds to the decrease of viscosity with temperature during the transition from glass to supercooled liquid. The peak in the loss modulus corresponds to the $\alpha$ relaxation, which is associated with the elastic to viscous transition phenomenon. Here, we focus on the $\alpha$ relaxation features of this metallic glass. (iii) At higher temperatures (above $760 \mathrm{~K}$ ), both $\mathrm{G}^{\prime}$ and $\mathrm{G}^{\prime \prime}$ exhibit a drastic increase, which corresponds to the crystallization process.

In order to further characterize the mechanical relaxations of the $\mathrm{Cu}_{46} \mathrm{Zr}_{46} \mathrm{Al}_{8}$ bulk metallic glass, isothermal experiments were performed at different temperatures. Fig.2 shows the frequency dependence of the normalized loss modulus $G^{\prime \prime} / G u$ of the $\mathrm{Cu}_{46} \mathrm{Zr}_{46} \mathrm{Al}_{8}$ bulk metallic glass at different temperatures. It can be seen that the loss modulus increases by decreasing the frequency at low isothermal temperature and the 
complete $\alpha$ relaxation peak can be observed at higher temperatures.

The Havriliak-Negami (HN) function [30] is used to describe the $\alpha$ relaxations in glassy materials. The $\mathrm{HN}$ equation can be expressed as:

$$
\mathrm{G}^{*}(\omega)=\frac{\Delta G}{\left[1+\left(i \omega \tau_{H N}\right)^{\alpha}\right]^{g}}
$$

where $\alpha$ and $g$ are the tow shape parameters determining the symmetric and asymmetric broadness of the loss modulus peak. $\Delta G$ is the relaxation strength, which is assumed as $\Delta \mathrm{G}=\mathrm{G}_{u}-\mathrm{G}_{r}\left(\mathrm{G}_{u}\right.$ denotes the unrelaxed modulus and $\mathrm{G}_{r}$ denotes the relaxed modulus). As shown in Fig. 2, the HN model is able to describe the shape of the $\alpha$ relaxation peak. The value of exponent $g$ was fixed at $g=0.45$. Fig. 3 shows the values of $\tau_{H N}, \alpha$ and $g$ obtained from the fitting of experimental data. The relaxation time follows a Vogel-Fulcher-Tamman behaviour above the glass transition, while it changes to an Arrhenius behaviour below $T_{g}$ with lower apparent activation energy. This change from supercooled liquid to glass dynamics is expected at the glass transition. The difference between as-cast and annealed samples is observed only below the glass transition, the as-cast samples show much shorter relaxation time due to higher amount of free volume frozen-in in the glass structure. The fictive temperatures of the two glassy states, defined as the temperature at which the glass behaviour intersects the supercooled liquid line, are specified in Fig. 3.

The values of $\alpha$ and $g$ are always between 0.3 and 0.4 (Fig. 3 (b)). This indicates that the shape of loss peak does not change significantly with temperature or glassy state. Here the value of $\alpha$ and $g$ corresponds to the exponent that determining the exponential decay of the high frequency wing of the loss modulus peak. This high-frequency exponent coincides with the Kholrausch-Williams-Watts exponent of the corresponding stress decay in the time domain [29]. As observed in other metallic glasses [31], the KWW exponent slightly increases with temperature at the glass transition.

Structural heterogeneity in metallic glasses have been confirmed by experimental and simulation techniques. In order to describe the dynamic mechanical properties of 
glassy materials with its microstructure, Perez et al. developed the quasi-point defect theory $[32,33]$. This theory assumes that glassy materials contain quasi-point defects, which are associated with the density fluctuation in nanoscale regions. These defects correspond to fluctuation of enthalpy or entropy. The existence of quasi-point defects has been verified by small angle X-ray scattering [34]. Due to these quasi-point defects, the atoms of metallic glass can perform relative motions, which results in relaxation behavior at macroscopic scale. In the framework of the quasi-point defects theory, the global characteristic relaxation time $\tau_{\text {relax }}$ that describes the mobility of atoms or molecules of the glassy material can be defined as [35]:

$$
\tau_{\text {relax }}=t_{0}\left(\frac{\tau_{\beta}}{t_{0}}\right)^{1 / \chi}
$$

where $\tau_{\beta}$ is the mean time of the thermally activated jump of a structural unit and follows the Arrhenius law, $t_{0}$ is a time scale parameter, $\chi$ is a correlation factor related to the quasi-point defect concentration $\left(C_{d}\right)$ ranging from 0 (full order-perfect crystal) to 1 (full disorder-perfect gas).

According to this model, the loss factor $\tan \delta$ of the glassy materials can be expressed as:

$$
\tan \delta=K_{0}\left(\omega \tau_{\text {relax }}\right)^{-\chi}
$$

Eq. (2) and (3) can be logarithm operated to eq. (4) and (5):

$$
\begin{gathered}
\ln \left(\tau_{\text {relax }}\right)=\ln t_{0}+\frac{1}{\chi} \ln \frac{\tau_{\beta}}{t_{0}} \\
\ln (\tan \delta)=\ln K_{0}-\chi\left(\ln \omega+\ln \tau_{\text {relax }}\right)
\end{gathered}
$$

The loss factor $\tan \delta$ can be expressed as [35]:

$$
\ln (\tan \delta)=-\chi \ln \omega-\chi \ln \left(\tau^{*}\right)+\ln K_{0}-\frac{U_{\beta}}{k T}
$$

where $\tau^{*}=t_{0}\left(\frac{\tau_{0 \beta}}{t_{0}}\right)^{1 / \chi}, U_{\beta}$ is the activation energy for the structural unit movement related to the $\beta$ relaxation and $k$ is the Boltzmann constant.

According to eq. (6), for each fixed temperature, the $\ln (\tan \delta)$ and $\ln \omega$ are in linear relationship and the slope of this linear relation is $\chi$.

Based on the eq. (6), 


$$
\left\{\frac{d \ln (\tan \delta)}{d \ln (\omega)}\right\}_{T}=-\chi(T)
$$

Fig.4 shows the variation of $\ln (\tan \delta)$ versus frequency of the $\mathrm{Cu}_{46} \mathrm{Zr}_{46} \mathrm{Al}_{8}$ bulk metallic glass at different temperatures. All the data can be well fitted by eq. (7). According to prediction of the quasi-point defect model, glassy materials remain in an iso-sconfigurational state when the temperature is below the glass transition temperature $T_{g}$. Thus, the correlation factor $\chi$ is constant. On the other hand, when the temperature rises above the $T_{g}$, a metastable thermodynamic equilibrium is reached and then concentration of quasi-point defects increases with temperature. Thus, the parameter $\chi$ is strongly dependent on temperature.

Fig.5 shows evolution of the correlation factor $\chi$ with temperature in the $\mathrm{Cu}_{46} \mathrm{Zr}_{46} \mathrm{Al}_{8}$ bulk metallic glass for both as-cast state and annealed state. It is evident from Fig.5 that the concentration of "defects" is almost constant when the temperature is below $T_{g}$, therefore the correlation factor $\chi$ remains nearly constant. When the temperature surpasses $T_{g}$, the correlation factor $\chi$ increases with elevated temperatures. The current experimental results are in good accordance with the prediction of the quasi-point defects model. In parallel, it should be noted that the correlation parameter $\chi$ of the annealed state is slightly lower than that of as-cast state. Many investigations have demonstrated that physical aging below $T_{g}$ reduces the concentration of defects, which induces a decrease of the atomic mobility. The results are in good agreement with the characteristic time of the aged sample, as shown in Fig.3 (a).

Dynamic mechanical tests were performed in MD simulations. Based on the definition of quasi-nearest atom (QNA), two atoms as a pair of QNA should meet the following three conditions [36]: (I) they are Voronoi nearest neighbor atoms of one specific atom; (II) they are adjacent to each other, defined by that their corresponding Voronoi faces of the Voronoi polyhedron centered by their common nearest neighbor share an edge; (III) they are not the Voronoi nearest neighbor atoms of each other. Therefore, the number of QNAs $\left(N_{Q}\right)$, connected to the defects of clusters, reflects the 
correlation between local structure and dynamic heterogeneity [37]. Fig. 6 shows the normalized storage modulus $E^{\prime} / E u$, averaged $N_{Q}\left(\left\langle N_{Q}\right\rangle\right)$ and normalized loss modulus $E " / E u$ as a function of temperature at different frequencies. Here, $E_{u}$ is the non-relaxed modulus, which is assumed to be $E^{\prime}$ at $100 \mathrm{~K}$. The frequencies were set to 1, 5, 10, 50 and $100 \mathrm{GHz}$. The isothermal temperature ranged from 100 to $1200 \mathrm{~K}$ with an interval of $100 \mathrm{~K}$. At low temperatures, both $E$ ' and $E$ "' change slightly with $T$. As temperature increases, $E$ ' drops sharply and $E$ " reaches peak values when the temperature rises to a certain critical value. Meanwhile, as shown in Fig. 6(a), the changing trend of $E$ ' is reverse to that of $\left\langle N_{Q}\right\rangle$. This suggests that adjusting of defects is inversely correlated to $E^{\prime}$. Fig. 6 also shows the influence of frequencies on the normalized storage modulus $E^{\prime}$ and normalized loss modulus $E$ ”. With the increase of frequency, the storage modulus $E^{\prime}$ and peaks of loss modulus $E$ " becomes higher. These results are consistent with related experiments $[4,5]$. The above results indicate that the defects of clusters have a strong correlation with $E^{\prime}$ and $E^{\prime \prime}$. Through adjusting the defects of clusters, dynamic heterogeneities in MGs can be changed.

To study the physical aging below $T_{g}$ of metallic glasses that reduces the concentration of defects and induces a decrease of the atomic mobility, the effects of physical aging on the width of the shear bands were investigated, as shown in Fig. 7. In the simulation, we define that the atoms with local shear strains higher than $20 \%$ constitute the shear band region [38]. It is noted that the shear bands narrowed down after the physical aging below $T_{g}$, which further confirmed that reducing the concentration of defects could induce a decrease of the atomic mobility. The structural rearrangement releases part of the free volume in metallic glasses with annealing, and liquid-like zones are transformed into solid-like zones that reducing the defects regions. Shear transformation zones occur preferentially in defects regions with lower critical shear stress. Less regions of defects correspond to the formation of less shear transformation zones, which leads to the formation of narrowed shear bands.

\section{Conclusion}

Dynamic mechanical relaxation behavior of $\mathrm{Cu}_{46} \mathrm{Zr}_{46} \mathrm{Al}_{8}$ metallic glass was 
investigated by mechanical spectroscopy. The main relaxation of the metallic glass was analysed by Havriliak-Negami (HN) model. The dynamic mechanical behavior of $\mathrm{Cu}_{46} \mathrm{Zr}_{46} \mathrm{Al}_{8}$ metallic glass can be describe well based on the quasi-point defects model. The correlated parameter $\chi$ of the annealed state is lower than that of as-cast state, which is related to a decrease of the atomic mobility. Dynamic mechanical tests performed in MD simulations showed that the changing trend of storage modulus $E$, is reverse to that of the QNAs number $\left\langle N_{Q}\right\rangle$, which suggests that adjusting of defects is inversely correlated to $E^{\prime}$. With the increase of frequency, the storage modulus $E^{\prime}$ and peaks of loss modulus $E$ ” becomes higher. Through adjusting the defects of clusters, dynamic heterogeneities in MGs can be changed. After the physical aging below $T_{g}$, the shear bands narrowed down notably, which further confirmed that reducing the concentration of defects could induce a decrease of the atomic mobility.

\section{Acknowledgement}

This work is partially supported by the NSFC (Grant Nos. 51401192, 51611130120 and 11572249), the Fundamental Research Funds for the Central Universities (No. 3102015ZY027), the Natural Science Foundation of Shaanxi Province (No. 2016JM5009) and the Aeronautical Science Foundation of China (2015ZF53072). 


\section{References}

[1] A. Inoue, Stabilization of metallic supercooled liquid and bulk amorphous alloys. Acta Mater. 48 (2000) 279-306.

[2] W.H. Wang, The elastic properties, elastic models and elastic perspectives of metallic glasses. Prog. Mater. Sci. 57 (2012) 487-656.

[3] A.L. Greer, Metallic glasses. Science 267 (1995) 1947-53.

[4] H.B. Yu, W.H. Wang, K. Samwer, The $\beta$ relaxation in metallic glasses: an overview. Mater. Today 16 (2013) 183-191.

[5] J.C. Qiao, J.M. Pelletier, Dynamic mechanical relaxation in bulk metallic glasses: A review. J. Mater. Sci. Technol. 30 (2014) 523-545.

[6] C. Liu, E. Pineda, D. Crespo, Mechanical relaxation of metallic glasses: An overview of experimental data and theoretical models. Metals 5 (2015) 1073-1111.

[7] K.L. Ngai, Relaxation and Diffusion in Complex Systems; Springer: New York; 2011.

[8] J.C. Qiao, R. Casalini, J.M. Pelletier, Y. Yao, Dynamics of the strong metallic glass $\mathrm{Zn}_{38} \mathrm{Mg}_{12} \mathrm{Ca}_{32} \mathrm{Yb}_{18}$. J. Non-Cryst. Solids 447 (2016) 85-90.

[9] Z. Wang, B.A. Sun, H.Y. Bai, W.H. Wang, Evolution of hidden localized flow during glass-to-liquid transition in metallic glass. Nat. Commun. 5 (2014) 5823.

[10] Q.J. Sun, L.N. Hu, C. Zhou, H.J. Zheng, Y.Z. Yue, Correlation between supercooled liquid relaxation and glass Poisson's ratio. J. Chem. Phys. 143 (2015) 164504.

[11] Q.J. Sun, C. Zhou, Y.Z. Yue, L.N. Hu, A direct link between the fragile-to-strong transition and relaxation in supercooled liquids. J. Phys. Chem. Lett. 5 (2014) 1170-1174.

[12] J.C. Qiao, J.M. Pelletier, R. Casalini, Relaxation of bulk metallic glasses studied by mechanical spectroscopy. J. Phys. Chem. B 117 (2013) 13658-13666. 
[13] J.M. Pelletier, D.V. Louzguine-Luzgin, S. Li, A. Inoue, Elastic and viscoelastic properties of glassy, quasicrystalline and crystalline phases in $\mathrm{Zr}_{65} \mathrm{Cu}_{5} \mathrm{Ni}_{10} \mathrm{Al}_{7.5} \mathrm{Pd}_{12.5}$ alloys. Acta Mater. 59 (2011) 2797-2806.

[14] E. Pineda, P. Bruna, B. Ruta, M. Gonzalez-Silveira, D. Crespo, Relaxation of rapidly quenched metallic glasses: Effect of the relaxation state on the slow, low temperature dynamics. Acta Mater. 61 (2013) 3002-3011.

[15] J.C. Qiao, Y.J. Wang, J.M. Pelletier, L.M. Keer, M.E. Fine, Y. Yao,

Characteristics of stress relaxation kinetics of $\mathrm{La}_{60} \mathrm{Ni}_{15} \mathrm{Al}_{25}$ bulk metallic glass. Acta Mater. 98 (2015) 43-50.

[16] Q. Wang, S.T. Zhang, Y. Yang, Y.D. Dong, C.T. Liu, J. Lu, Unusual fast secondary relaxation in metallic glass. Nature Commun. 6(2015) 7876

[17] L.Z. Zhao, R.J. Xue, Z.G. Zhu, K.L. Ngai, W.H. Wang, H.Y. Bai, A fast dynamic mode in rare earth based glasses. J. Chem. Phys. 144 (2016) 204507.

[18] H.B. Yu, X. Shen, Z. Wang, L. Gu, W.H. Wang, H.Y. Bai, Tensile plasticity in metallic glasses with pronounced $\beta$ relaxation. Phys. Rev. Lett. 108 (2012) 015504.

[19] H.B. Yu, W.H. Wang, H.Y. Bai, Y. Wu, M.W. Chen, Relating activation of shear transformation zones to $\beta$ relaxations in metallic glasses. Phys. Rev. B 81 (2010) 220201.

[20] H.B. Yu, K. Samwer, Y. Wu, W.H. Wang, Correlation between $\beta$ relaxation and self-diffusion of the smallest constituting atoms in metallic glasses. Phys. Rev. Lett. 109 (2012) 095508.

[21] H.B. Yu, K. Samwer, W.H. Wang, H.Y. Bai, Chemical influence on $\beta$-relaxations and the formation of molecule-like metallic glasses. Nat. Commun. 4 (2013) 2204.

[22] J.C. Qiao, Y. Yao, J.M. Pelletier, L.M. Keer, Understanding of micro-alloying on plasticity in $\mathrm{Cu}_{46} \mathrm{Zr}_{47-\mathrm{x}} \mathrm{Al}_{7} \mathrm{Dy}_{\mathrm{x}}(0 \leq \mathrm{x} \leq 8)$ bulk metallic glasses under compression: Based on mechanical relaxations and theoretical analysis. Inter. J. Plast. 82 (2016) $62-75$

[23] S. Etienne, J.Y. Cavaillé, J. Perez, R. Point, M. Salvia, Automatic system for analysis of micromechanical properties. Rev. Sci. Inst. 53 (1982) 1261-1266. 
[24] S. Plimpton. Fast parallel algorithms for short-range molecular dynamics, J. Comput. Phys. 117 (1995) 1-19.

[25] Y.Q. Cheng, E. Ma, H.W. Sheng. Atomic level structure in multicomponent bulk metallic glass, Phys. Rev. Lett. 102 (2009) 245501.

[26] H.B. Yu, K. Samwer. Atomic mechanism of internal friction in a model metallic glass. Phys. Rev. B. 90 (2014) 144201.

[27] J.M. Pelletier, D. V. Louzguine-Luzgin, S. Li, A. Inoue. Elastic and viscoelastic properties of glassy, quasicrystalline and crystalline phases in $\mathrm{Zr}_{65} \mathrm{Cu}_{5} \mathrm{Ni}_{10} \mathrm{Al}_{7.5} \mathrm{Pd}_{12.5}$ alloys. Acta Mater. 59 (2011) 2797-2806.

[28] J.C. Qiao, J.M. Pelletier, C. Esnouf, Y. Liu, H. Kato. Impact of the structural state on the mechanical properties in a $\mathrm{Zr}-\mathrm{Co}-\mathrm{Al}$ bulk metallic glass. J. Alloy. Compd. 607 (2014) 139-149.

[29] Z.F. Yao, J.C. Qiao, J.M. Pelletier, Y. Yao. Characterization and modeling of dynamic relaxation of a Zr-based bulk metallic glass. J. Alloy. Compd. 690 (2017) 212-220.

[30] S. Havriliak. A complex plane representation of dielectric and mechanical relaxation processes in some polymers. Polymer 8 (1967) 161-210.

[31] Z. Wang, B.A. Sun, H.Y. Bai, W.H. Wang. Evolution of hidden localized flow during glass-to-liquid transition in metallic glass. Nat. Commun. 5 (2014) 5823.

[32] J. Perez, Quasi-punctual defects in vitreous solids and liquid-glass transition, Solid State Ionics 39 (1990) 69-79.

[33] J. Perez, S. Etienne, J. Tatibouet, Determination of glass-transition temperature by internal-friction measurements, Phys. Status Solidi A 121 (1990) 129-138.

[34] C. Gauthier, J.M. Pelletier, L. David, G, Vigier, J. Perez, Relaxation of non-crystalline solids under mechanical stress, J. Non-Cryst. Solids 274 (2000) $181-187$.

[35] J.C. Qiao, J.M. Pelletier, Mechanical relaxation in a Zr-based bulk metallic glass: analysis based on physical models, J. Appl. Phys. 112 (2012) 033518.

[36] S.P. Pan, S.D. Feng, J.W. Qiao, W. M. Wang, J. Y. Qin. Correlation between local 
structure and dynamic heterogeneity in a metallic glass-forming liquid. J. Alloys Compd. 664 (2016) 65-70.

[37] S.D. Feng, K.C. Chan, S.H. Chen, L. Zhao, R.P. Liu. The role of configurational disorder on plastic and dynamic deformation in $\mathrm{Cu}_{64} \mathrm{Zr}_{36}$ metallic glasses: A molecular dynamics analysis, Sci. Rep. 7(2017) 40969.

[38] Z.D. Sha, Q.X. Pei, V. Sorkin, P.S. Branicio, Y.W. Zhang, H.J. Gao. On the notch sensitivity of CuZr metallic glasses. Appl. Phys. Lett. 103 (2013) 081903. 


\section{Figure captions}

Fig. 1. Normalized storage $\left(G^{\prime}\right)$ and loss modulus $\left(G^{\prime \prime}\right)$ of the $\mathrm{Cu}_{46} \mathrm{Zr}_{46} \mathrm{Al}_{8}$ bulk metallic glass as a function of temperature (heating rate is $3 \mathrm{~K} / \mathrm{min}$ ).

Fig. 2. Evolution of the normalized loss modulus $G^{\prime \prime} / G u$ as a function of frequency at different temperatures for $\mathrm{Cu}_{46} \mathrm{Zr}_{46} \mathrm{Al}_{8}$ bulk metallic glass: (a) As-cast state; (b) Annealed state (the metallic glass was aged at $623 \mathrm{~K}$ for 16 hours). Solid lines are fittings to a Havriliak-Negami (HN) function.

Fig. 3. Temperature dependence of (a) $\tau_{H N}, \alpha g$ (b) as-quenched (blue dots) and relaxed (red dots) amorphous ribbons obtained from fitting of the experimental data to the HN function of Eq. (1).

Fig.4. Influence of the driving frequency on the loss factor at different temperatures in the $\mathrm{Cu}_{46} \mathrm{Zr}_{46} \mathrm{Al}_{8}$ bulk metallic glass (the isothermal temperature ranges from 638 to $718 \mathrm{~K}$ with an interval of $5 \mathrm{~K}$ ). Solid lines are fitted by Eq. (7).

Fig.5. Evolution of the correlation factor $\chi$ with temperature for $\mathrm{Cu}_{46} \mathrm{Zr}_{46} \mathrm{Al}_{8}$ bulk metallic glass.

Fig. 6. Evolution of (a) normalized storage modulus $E^{\prime} / E u$, averaged $N_{Q}\left(\left\langle N_{Q}\right\rangle\right)$ and (b) normalized loss modulus $E " / E u$ as a function of temperature at different frequencies for $\mathrm{Cu}_{46} \mathrm{Zr}_{46} \mathrm{Al}_{8}$ metallic glass.

Fig. 7. The width of shear band showed by local shear strain of $\mathrm{Cu}_{46} \mathrm{Zr}_{46} \mathrm{Al}_{8}$ metallic glass models when the total strain is $7 \%$ at different state: (a) As-cast state; (b) Annealed state (the metallic glass has been aged at $650 \mathrm{~K}$ with aging time of $5 \mathrm{~ns}$ ). 


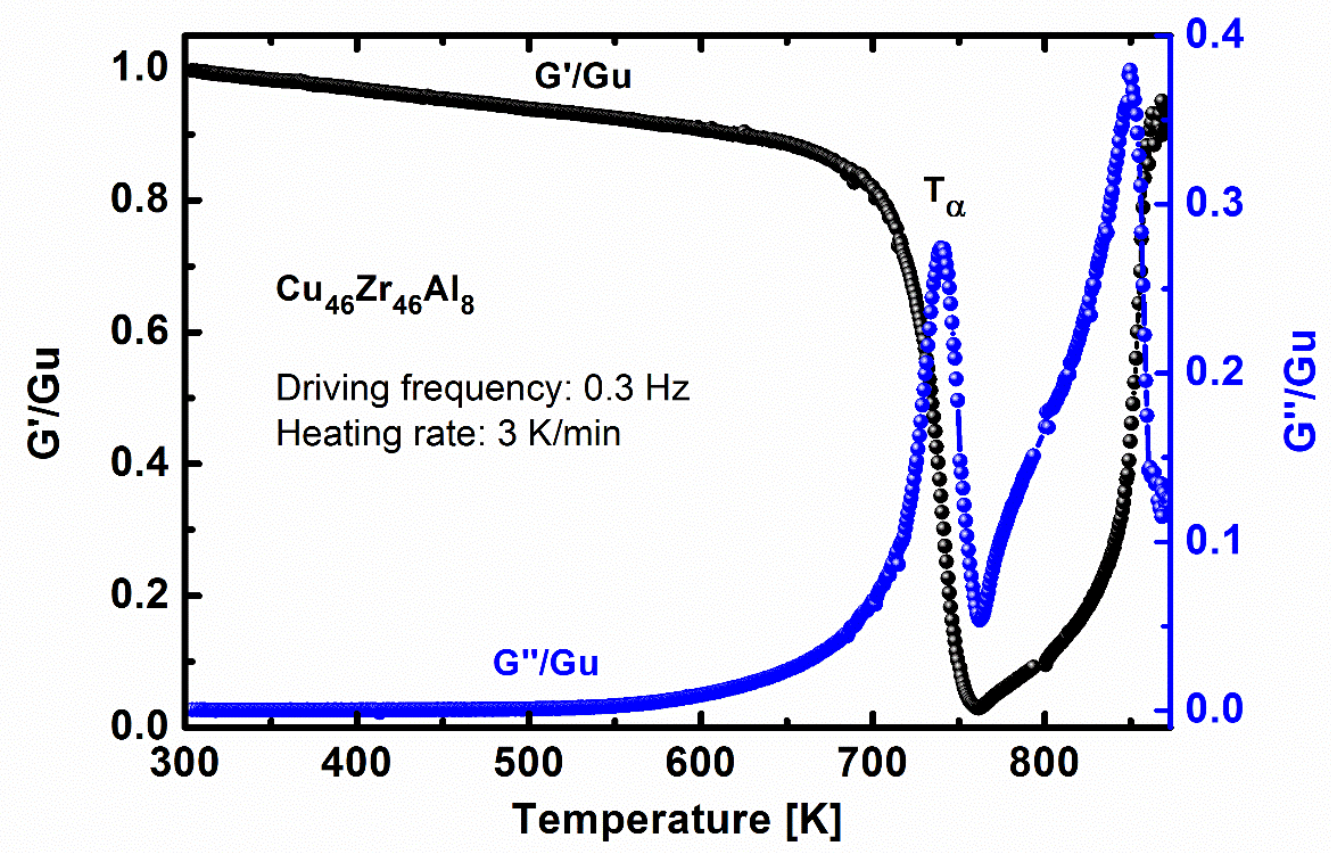

Fig. 1 


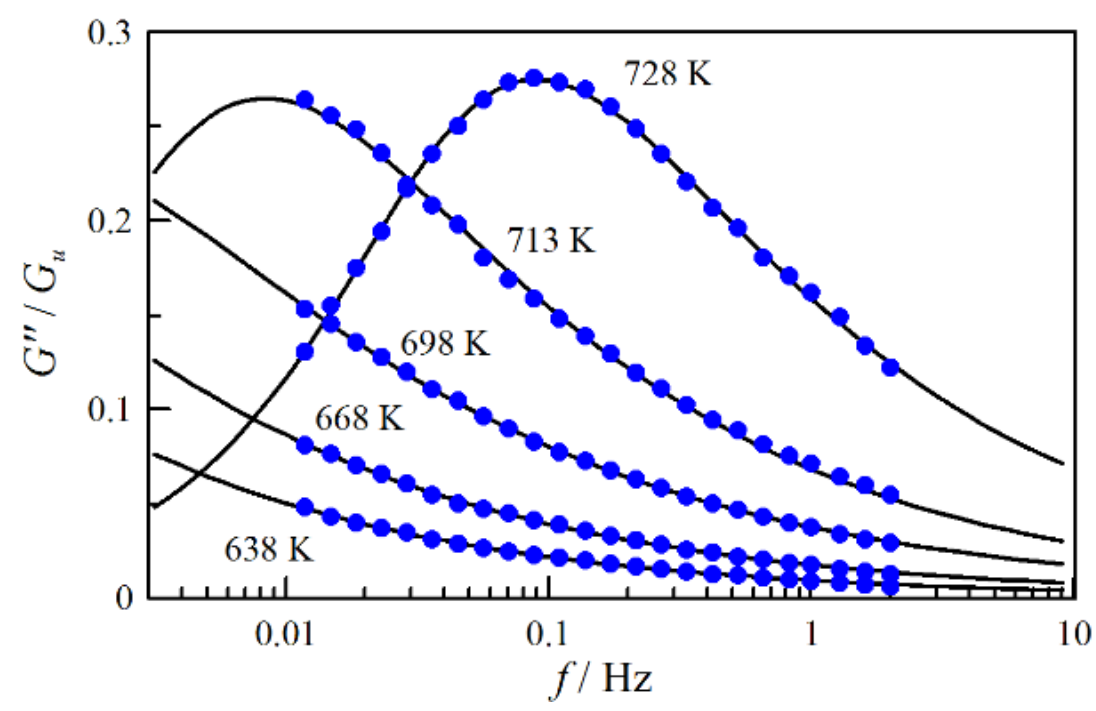

(a)

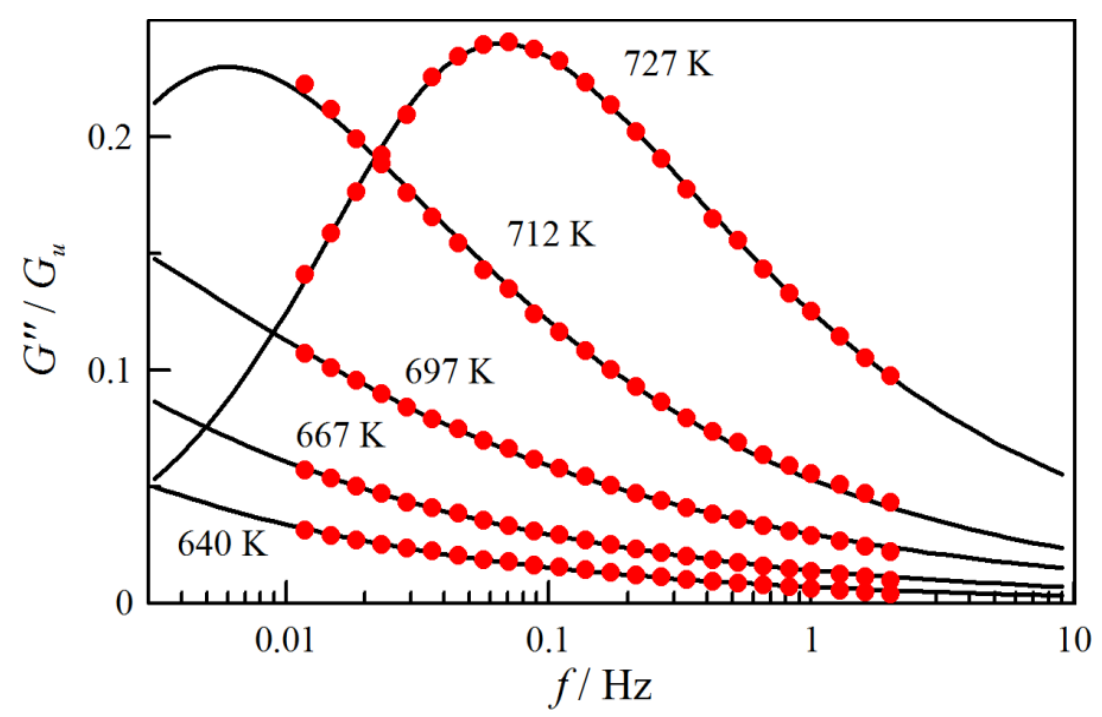

(b)

Fig. 2 


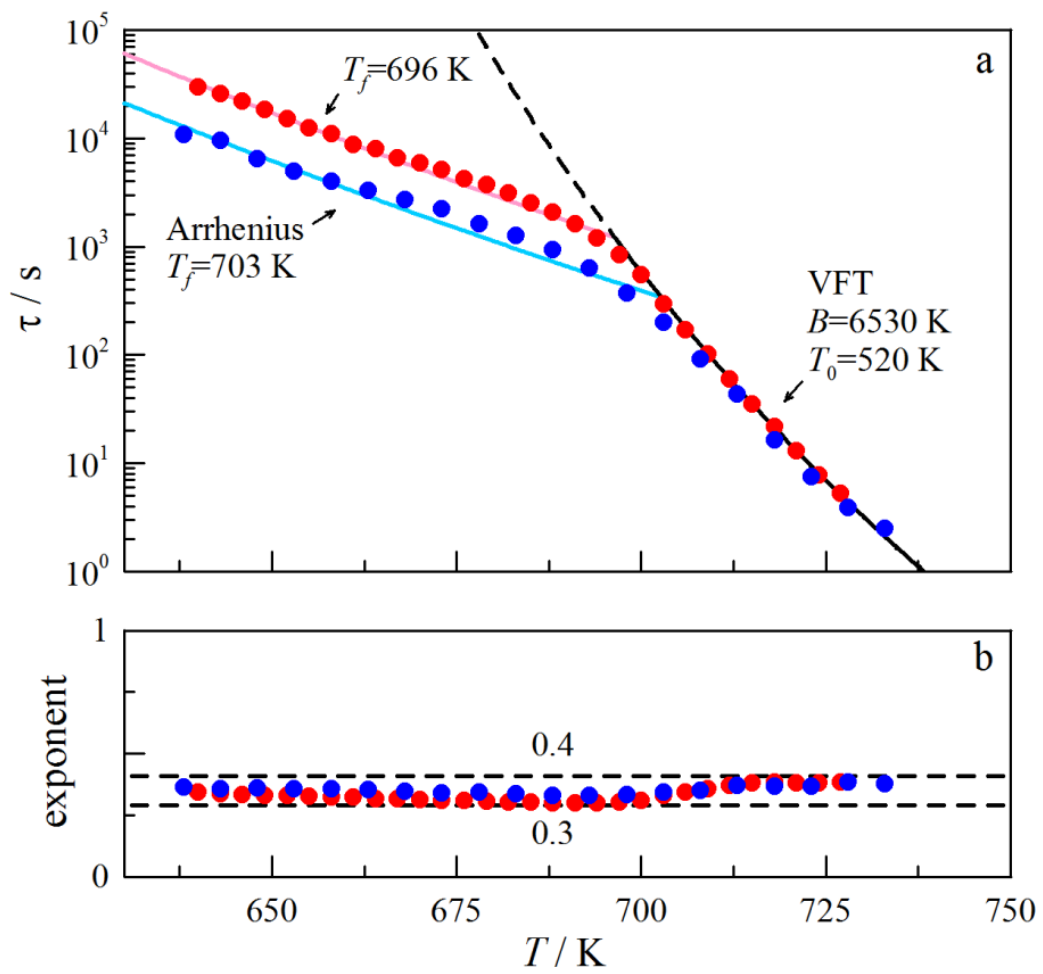

Fig. 3 


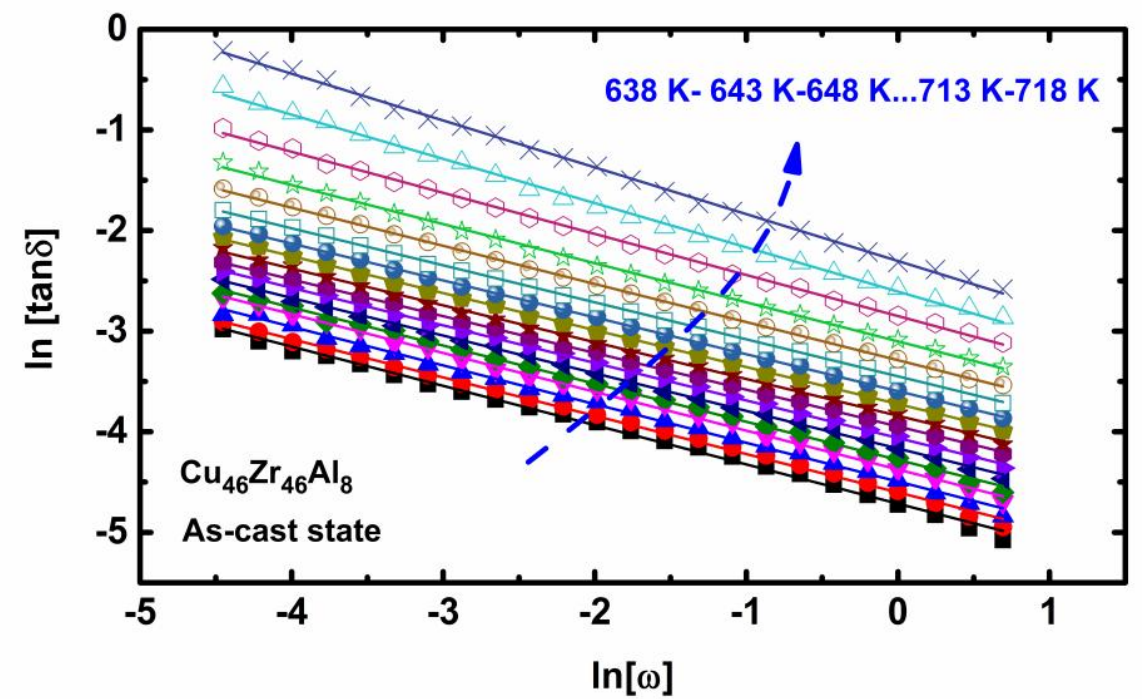

Fig. 4 


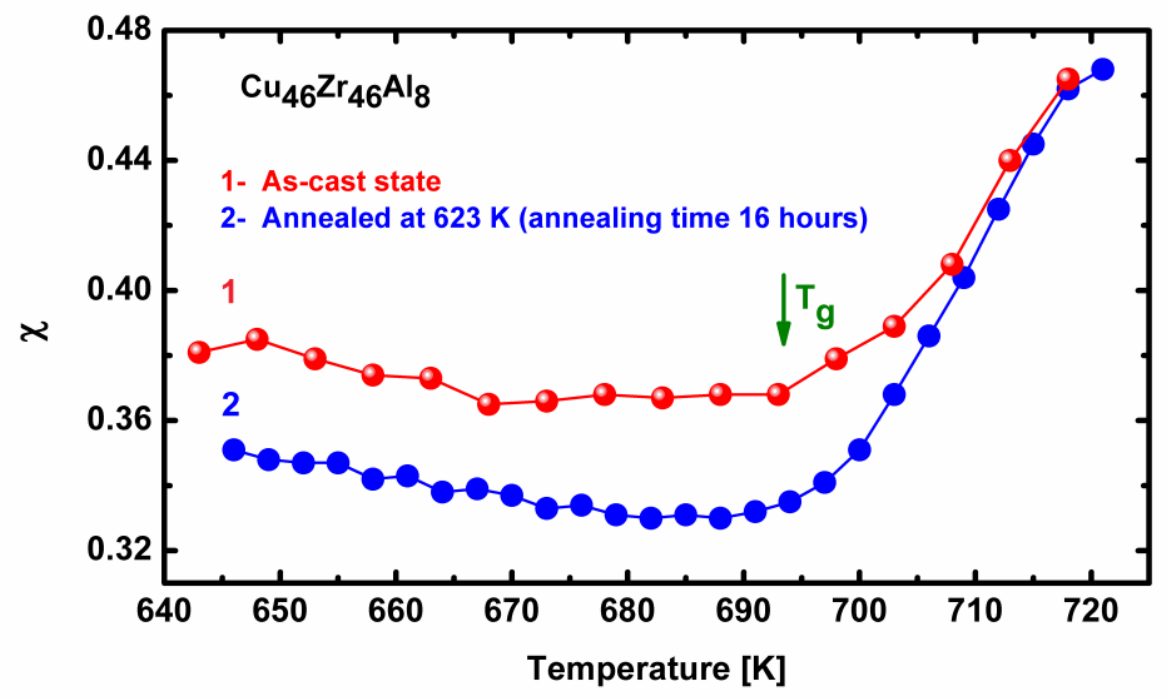

Fig. 5 

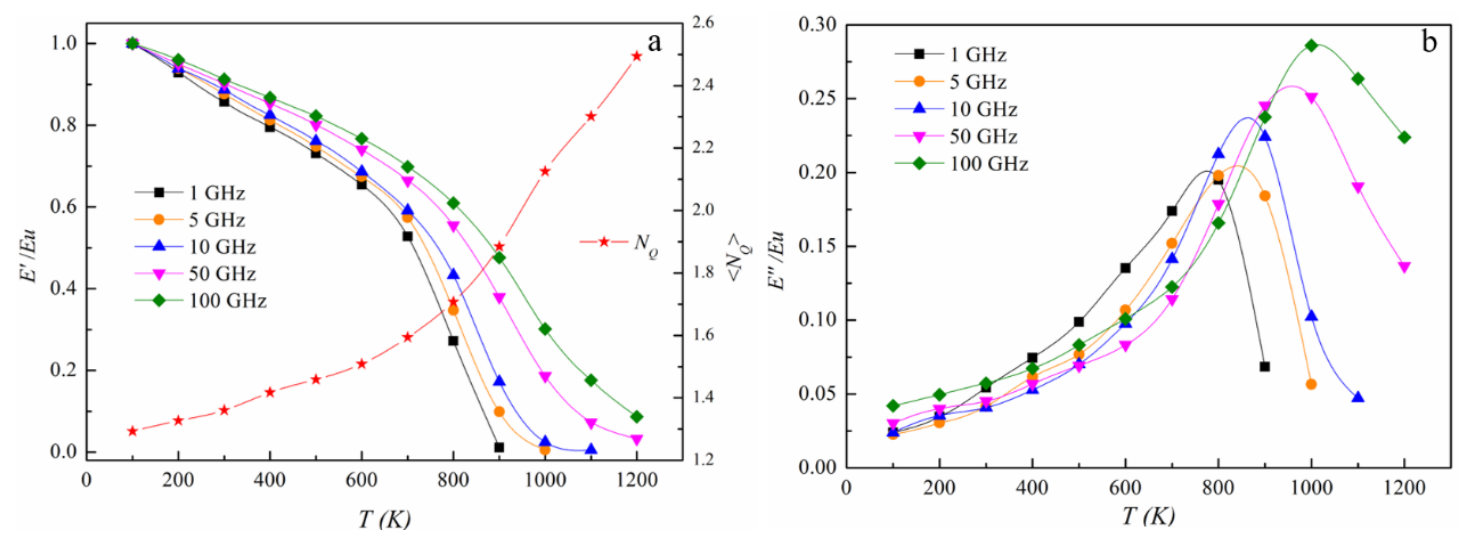

Fig. 6 


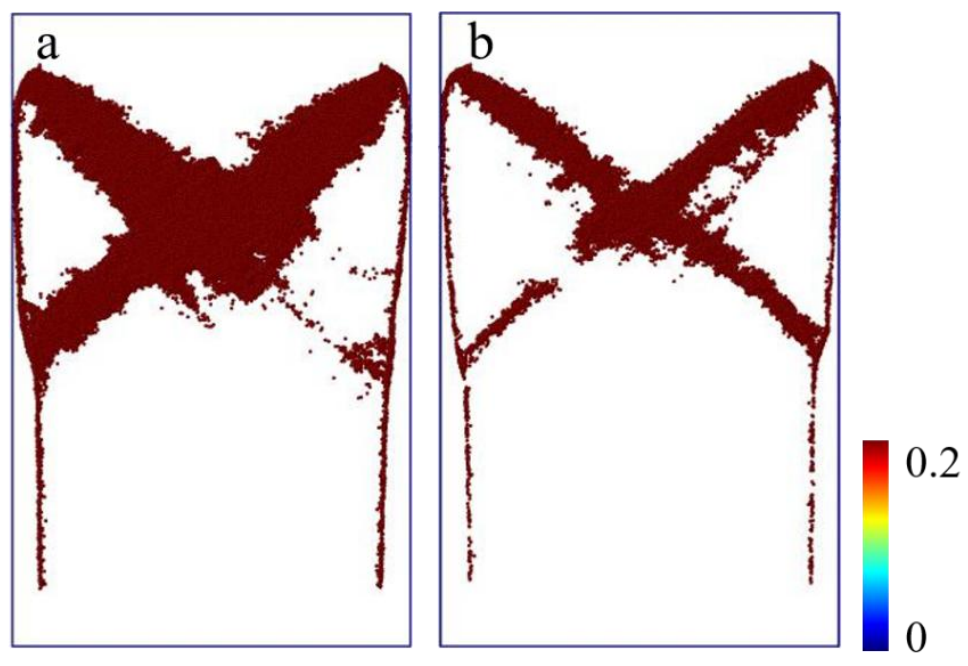

Fig. 7 